\title{
Solid Circle-Packings in the Euclidean Plane
}

\author{
A. Heppes \\ Computer and Automation Institute, Hungarian Academy of Science, \\ Budapest, Hungary
}

To L. Fejes Tóth on his seventy-fifth birthday

\begin{abstract}
The main result of this paper is the proof of a conjecture of $\mathrm{L}$. Fejes Tóth saying that the incircles of the Archimedean tiling $(4,8,8)$ form a solid packing. To achieve this a new technique, the method of weighted densities, has been developed and applied, besides the case mentioned, to prove the solidity of a number of other circle-packings in the Euclidean plane.
\end{abstract}

\section{Introduction}

A packing of convex discs is said to be solid if no finite subset of the discs can be rearranged so as to obtain a packing not congruent to the original one. According to a longstanding conjecture the incircles of the tiles of any (spherical, Euclidean, or hyperbolic) trihedral Archimedean tiling form a solid packing [3]. This has been proved for some spherical tilings and for many hyperbolic tilings (see [2] and [1]), but, in the particularly interesting case of the Euclidean tiling $(4,8,8)$, the conjecture resisted all attempts of proving it. In this paper we develop a method that enables us to confirm the conjecture for the two previously unsettled Euclidean tilings $(4,8,8)$ and $(4,6,12)$ and to prove the solidity of some other circle-packings.

\section{A General Theorem}

In order to phrase our main result we define some notions.

We consider compact packings of circles (open circular discs) in which every circle $c$ is tangent to several other circles, say $c_{1}, \ldots, c_{n}, c_{n+1}=c_{1}$, such that $c_{i}$ is tangent to $c_{i+1}, i=1, \ldots, n$, and the convex hull of the centers of the neighbors 
contains the center of $c$ [4]. Such a packing of circles generates a trigonal tiling whose edges are the segments connecting the centers of circles in contact. Keeping in mind that each tile contains three circular sectors, we say that the above packing generates a marked trigonal tiling [7].

Again, we say that a marked tiling is uniquely determined if no finite subset of the tiles can be replaced by a set of marked tiles each congruent to one tile of the original tiling so as to obtain a "properly marked" tiling not congruent to the original one. In our case the term "properly marked" means that the circular sectors fit together forming complete circles.

Let $t$ be a triangle spanned by the centers $O_{1}, O_{2}, O_{3}$ of three circles of radii $r_{1}$, $r_{2}, r_{3}$. We assign to the circle centered at $O_{i}$ a positive weight $w\left(r_{i}\right)$ and define the function

$$
\delta=\frac{\frac{1}{2} \cdot \sum_{i=1}^{3} r_{i}^{2} \cdot \alpha_{i} \cdot w\left(r_{i}\right)}{A}
$$

where $\alpha_{i}$ is the angle of $t$ at $O_{i}$ and $A$ denotes the area of $t(i=1,2,3)$. We consider only normal triples of circles and normal triangles associated with them defined by the property that no circle intersects the opposite side of the triangle. Therefore it is reasonable to call $\delta$ the density of the circles in $t$ or, in short, the density of $t$. A normal triangle is called tight (stretched) if the circles are mutually tangent (if one circle is tangent to the other two and to the opposite side) (Fig. 1).

Now we can formulate the following:

Theorem. A compact packing of circles of radii $r_{1}, \ldots, r_{k}$ is solid if the following two conditions are satisfied:

(i) Positive weights $w(r)_{i}$ can be assigned to the circles of radius $r_{i}(i=1, \ldots, k)$ in such a way that in the marked tiling generated by the packing all tiles have equal densities, while the density of any other tight triangle associated with a triple of circles of radii $r_{1}, \ldots, r_{k}$ is smaller.

(ii) The marked tiling generated by the packing is uniquely determined.
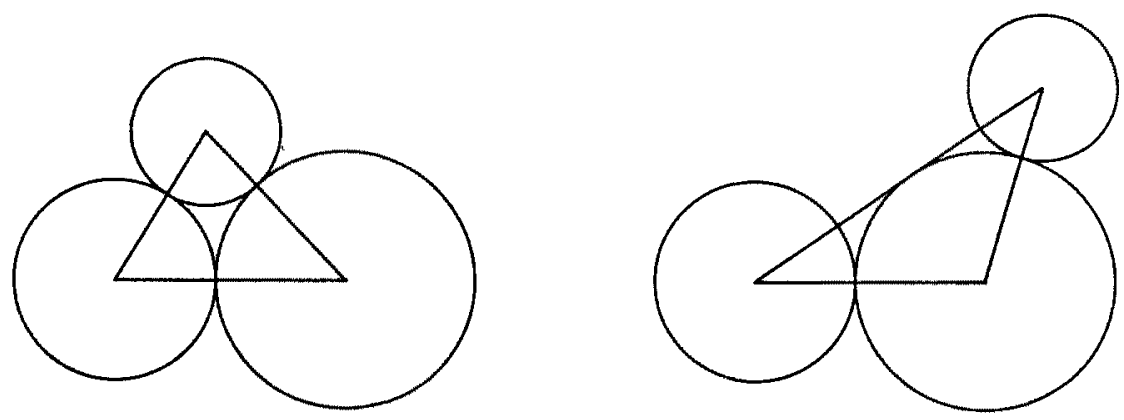

Fig. 1. Tight and stretched triples. 
To generalize the notion of solidity we say that a packing of convex discs is weakly solid if for any finite subset of the discs there are but a finite number of ways to rearrange the discs of the subset to obtain a packing. Using this notion we can complement the theorem with the following:

Remark. If the hypotheses of the theorem are reduced by removing condition (ii). then the packing is only weakly solid.

It will also be shown that these two conditions are independent: none of them implies the other.

\section{Preparations}

Definitions. By a normal decomposition of a packing of circles we mean the decomposition of the plane into normal triangles. A function $f(x)$ is called (strictly) quasi-convex in an interval if for any given subinterval of this interval $f(x)$ attains its maximum at one or both ends of the interval (only).

First we prove the following:

Lemma 1. Let the radii $r_{i}$ and positive weights $w\left(r_{i}\right)(i=1, \ldots, k)$ be given. We consider all normal triangles associated with a triple of circles whose radii belong to the set $\left(r_{1}, \ldots, r_{k}\right)$. Then each triangle of maximal density is either tight or stretched.

The proof of Lemma 1 is based on the following result of Hárs in [6]:

Lemma 2. Let $\alpha_{i}, \alpha_{j}, \alpha_{k}$ be the angles and let $A$ be the area of a triangle. If we fix the length of the sides opposite to $\alpha_{i}$ and $\alpha_{j}$, then, for arbitrarily given positive weights $w_{i}$, $w_{j}$, and $w_{k}$, the function

$$
\vartheta\left(\alpha_{k}\right)=\frac{\alpha_{i} w_{i}+\alpha_{j} w_{j}+\alpha_{k} w_{k}}{A}
$$

is strictly quasi-convex in $(0, \pi)$.

Proof of Lemma 1. Clearly, for weights $w_{s}=\frac{1}{2} \cdot r_{s}^{2} \cdot w\left(r_{s}\right)(s=i, j, k)$, the density of a triangle and its weighted "angle-density" $9\left(\alpha_{k}\right)$ coincide. As in a large triangle the density is small, when looking for the densest arrangement it is enough to consider normal triangles of restricted size. However, the set of these triangles is compact, thus the existence of a triangle of maximal density follows easily. Therefore, it is sufficient to show that any normal triangle that is neither tight nor stretched is not one of greatest density.

We consider a normal triangle that is neither tight nor stretched and distinguish two cases. 
Case 1. No circle is tangent to the opposite side of the triangle (consequently it is not stretched) and there are two circles, say the first and the second, that are not tangent (thus the triangle is not tight either). Let us apply Lemma 2 using the substitution suggested above:

$$
\begin{aligned}
& w_{1}=\frac{\rho_{1}^{2} \cdot w\left(\rho_{1}\right)}{2}, \\
& w_{2}=\frac{\rho_{2}^{2} \cdot w\left(\rho_{2}\right)}{2}, \\
& w_{3}=\frac{\rho_{3}^{2} \cdot w\left(\rho_{3}\right)}{2},
\end{aligned}
$$

where $\rho_{j}$ denotes the actual values of the radii $(j=1,2,3)$ and let $\beta$ and $\gamma$ be those values of angle $\alpha_{3}$ for which the triangle stops being normal, or, in other words, where an additional contact occurs (Fig. 2(a)). According to Lemma 2 the weighted angle-density cannot attain its maximum for an angle $\alpha_{3}$ lying strictly between $\beta$ and $\gamma$. Thus, the triangle in question is not extremal.

Case 2. One circle, say the third one, is tangent to the opposite side $\mathrm{O}_{1} \mathrm{O}_{2}$ of the triangle (thus the triangle is not tight), and this circle does not touch both of the other circles, say the first and the third are not tangent (therefore it is not stretched). Let $O_{3}^{\prime}$ be the reflection of $O_{3}$ in straight line $O_{1} O_{2}$ (Fig. 2(b)). Clearly, both isosceles triangles $\mathrm{O}_{1} \mathrm{O}_{3} \mathrm{O}_{3}^{\prime}$ and $\mathrm{O}_{2} \mathrm{O}_{3} \mathrm{O}_{3}^{\prime}$ are normal, and, for the densities $\delta_{0}$, $\delta_{1}, \delta_{2}$ of the triangles $\mathrm{O}_{1} \mathrm{O}_{2} \mathrm{O}_{3}, \mathrm{O}_{1} \mathrm{O}_{3} \mathrm{O}_{3}^{\prime}, \mathrm{O}_{2} \mathrm{O}_{3} \mathrm{O}_{3}^{\prime}$,

$$
A_{1} \delta_{1}+A_{2} \delta_{2}=2 \cdot A \cdot \delta_{0}=\left(A_{1}+A_{2}\right) \cdot \delta_{0}
$$

holds, where $A_{i}$ denotes the area of $O_{i} O_{3} O_{3}^{\prime}(i=1,2)$. Consequently, $\delta_{0}$ cannot be the maximum of $\delta$ except for $\delta_{1}=\delta_{2}=\delta_{0}$. But, since $O_{1} O_{3} O_{3}^{\prime}$ belongs to Case 1 , neither this triangle nor $\mathrm{O}_{1} \mathrm{O}_{2} \mathrm{O}_{3}$ can be of maximal density.

This completes the proof of Lemma 1.

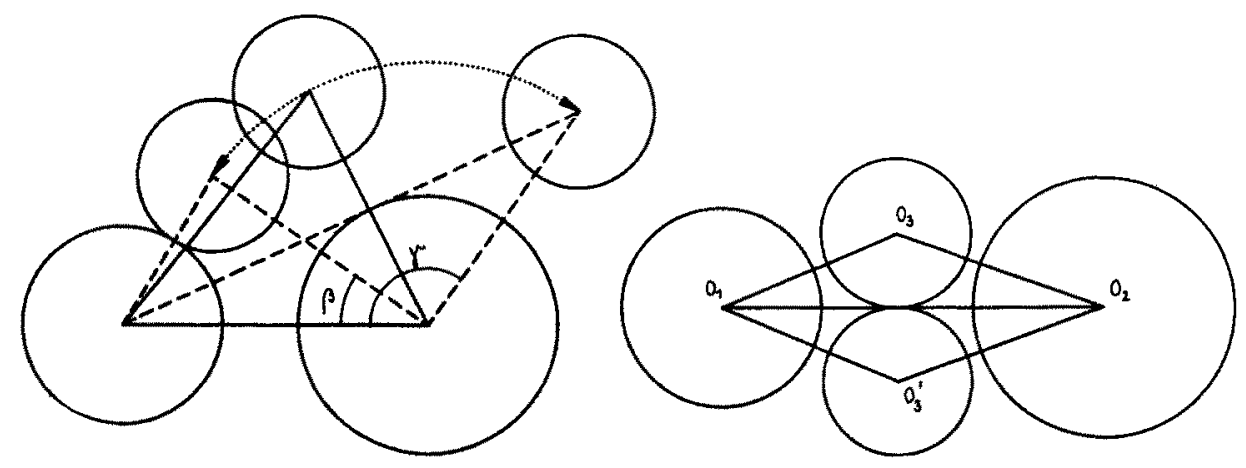

Fig. 2 
Remark. Applying the same reflection we used in the discussion of Case 2 it is easy to see that whenever the maximal density is attained by a stretched triangle there exists at least one tight triangle of the same density. Consequently, in order to find the maximal density for a given set of radii $\rho_{i}$ and the corresponding weights $w\left(\rho_{i}\right)$ it is enough to compare the densities for the $k+2 \cdot\left(\begin{array}{l}k \\ 2\end{array}\right)+\left(\begin{array}{l}k \\ 3\end{array}\right)$ tight triangles.

To prepare the proof of the Theorem we rephrase a result of Fejes Tóth and Molnár [5]:

Lemma 3. To any saturated packing of circles of radii $\geq \rho>0$ there exists $a$ normal decomposition-even so that each segment connecting the centers of tangent circles is a side of a triangle of the decomposition.

A packing of circles of radii $\geq \rho$ is called saturated if there is no room left for a further circle of radius $\rho$ without overlapping.

\section{The Proof of the Theorem}

Let $P$ be a compact packing for which condition (i) is valid, let $S$ be an arbitrary set of $n>0$ circles of $P$, and let $S^{\prime}$ be a rearrangement of these circles that together with the rest $P-S$ of the packing forms a new packing $P^{\prime}$. We show that $P^{\prime}$ can be decomposed into normal triangles so that this decomposition coincides with the marked trigonal tiling generated by $\boldsymbol{P}$ except for a finite set of triangles depending only on the choice of the subset $S$, and all triangles of the decomposition are tight. Thus, since the number of such decompositions is finite, the packing is weakly solid. If condition (ii) also holds, then there is only one such decomposition, consequently the packing is solid.

Let $U$ be the union of a finite number of triangles of the decomposition of $P$ that covers $S$ and $S^{\prime}$ as well. Now we define a weighted packing problem for $U$. We consider all sets of circles of radius $r_{1}, \ldots, r_{k}$ that

completely lie in $U$,

together with $P-S$ form a packing,

maximize the density of such a packing within $U$, all circles of radius $r_{i}$ taken with the weights $w\left(r_{i}\right)$ defined in condition (i).

Clearly, the original set $S$ provides an extremal solution since $U$ can be decomposed into tight triangles each maximizing the (weighted) density. The contribution of $S$ and $S^{\prime}$ is the same to the density in $U$, thus $P^{\prime}$ is also extremal, consequently it is saturated. Then-by Lemma $3-P^{\prime}$ can be decomposed into normal triangles in such a way that the boundary of $U$ (consisting of segments each connecting the centers of a pair of circles in contact) is not "crossed" by triangles, i.e., $U$ itself is the union of a finite set of these triangles. Thus, the decompositions of the two packings coincide outside $U$. 
The equality of the contributions mentioned above also implies that each triangle of this second decomposition of $U$ maximizes the weighted density. Then, by Lemma 1, each of these triangles is either tight or stretched. In fact none of them is stretched because stretched triangles could occur in $P^{\prime}$ only in symmetrical pairs, implying the existence of touching pairs of circles, the centers of which are not connected by a side of a triangle. But this would contradict the basic property of the second decomposition based on Lemma 3. Thus, the decomposition of $P^{\prime}$ consists of tight triangles.

This completes the proof of the Theorem.

\section{Applications}

Verifying condition (i) for a particular compact packing is just a matter of finding a solution for a system of linear inequalities. If a solution exists, the packing is weakly solid. Its (full) solidity, however, depends on the fact of whether the corresponding marked tiling is uniquely determined. In the simple cases discussed below, answering this question is no more than an exercise either, thus, except for some sample cases, the proofs are not discussed in detail.

First the cases of the Archimedean packings previously mentioned are discussed, then some regular packings with two-dimensional perodicity and some families of packings having only one-dimensional periodicity are discussed. Finally, the existence of solid packings without perodicity is illustrated.

Each of the compact packings considered in the following sections consists of circles of two or three different sizes. For notational simplicity we assume that $r_{1}=1>r_{2}>r_{3}$ holds. The density in the tight triangle $t_{i j k}$ generated by circles of radii $r_{i}, r_{j}, r_{k}$ is denoted by $d_{i j k}$.

\subsection{Archimedean Packings}

Let $A_{1}, A_{2}$, and $A_{3}$ be the packings obtained by inscribing circles into the tiles of $(3,12,12),(4,8,8)$, and $(4,6,12)$, respectively (Fig. 3$)$. (The solidity of packing $A_{1}$ can be (and has been long ago) settled with the following argument: Let $S$ be a solid packing of circles and let $c$ be the largest circle that fits into the gaps of $S$. If the
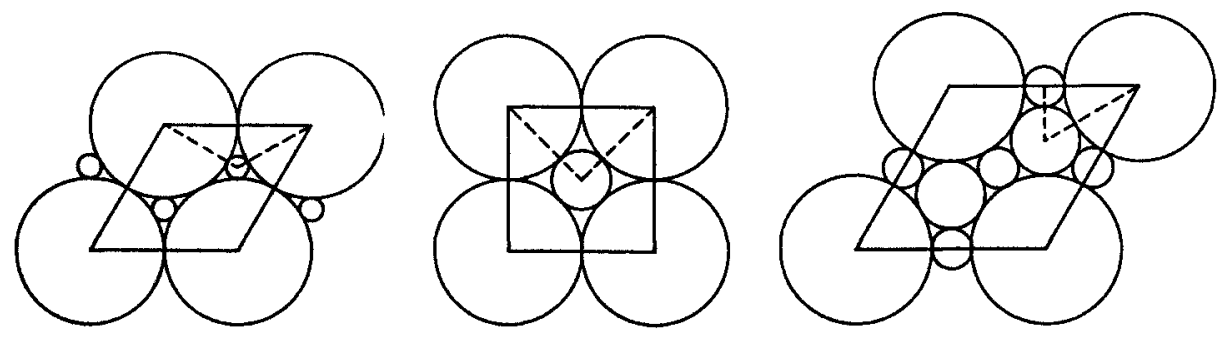

Fig. 3. Archimedean packings $A_{1}, A_{2}$, and $A_{3}$. 
Table 1

\begin{tabular}{|c|c|c|}
\hline & $A_{1}$ & $A_{2}$ \\
\hline$r_{2}$ & $=2 / \sqrt{3}-1=0.154 \ldots$ & $=\sqrt{2}-1=0.4142 \ldots$ \\
\hline$w\left(r_{i}\right)$ & $=1$ & $=1$. \\
\hline$w\left(r_{2}\right)$ & $=0.9$ & $=0.95$ \\
\hline$d_{111}$ & $=0.90689 \ldots$ & $=0.90689 \ldots$ \\
\hline$d_{112}$ & $=0.94596 \ldots{ }^{*}$ & $=0.91341 \ldots *$ \\
\hline$d_{122}$ & $=0.93388 \ldots$ & $=0.90132 \ldots$ \\
\hline$d_{222}$ & $=0.81620 \ldots$ & $=0.86155 \ldots$ \\
\hline
\end{tabular}

different "possible" locations of $c$ are disjoint, then the packing $S$ ' generated from $S$ by inscribing copies of $c$ into all sufficiently large gaps of $S$ is solid.)

The corresponding radii, assigned weights, and the (weighted) densities $d_{i j k}$ are shown in Tables 1 and 3. This shows that in each case condition (i) is fulfilled. (In each column of the tables the rows of maximal density are marked by ${ }^{*}$ ).

In each of these packings there exists only one tight triangle of maximal density and, when refilling the hole $U$ properly, the position of the next tile along each boundary segment is clearly unique, thus the corresponding marked tiling is uniquely determined (condition (ii)).

\subsection{Regular Packings}

A packing of circles is called regular if to any pair of circles of the same size there exists a congruency that maps the whole packing onto itself while one circle of the selected pair is mapped onto the other one.

To define a particular regular compact packing it is enough to describe the ring of the neighbors around a circle of each size in the packing. Most of these packings are well known as candidates for the title "densest packing of circles of given homogeneity" (see, e.g., [5] or [8]). The homogeneity of a packing of circles is the greatest lower bound for the ratio of any pair of radii in the packing. Of the packings discussed below, $R_{4}, \ldots, R_{7}$ consist of two kinds of circles while three sizes are used in $R_{8}$.

The assigned weights and the weighted densities in all tight triangles are given in Tables 2 and 3 . The existence of weight systems for which a certain pair of densities

Table 2

\begin{tabular}{lllll}
\hline & \multicolumn{1}{c}{$\boldsymbol{R}_{4}$} & \multicolumn{1}{c}{$\boldsymbol{R}_{5}$} & \multicolumn{1}{c}{$\boldsymbol{R}_{6}$} & \multicolumn{1}{c}{$\boldsymbol{R}_{7}$} \\
\hline$r_{2}$ & $=0.6375 \ldots$ & $=0.53329 \ldots$ & $=0.34919 \ldots$ & $=0.28077 \ldots$ \\
$w\left(r_{1}\right)$ & $=1$. & $=1$. & $=1$. & $=1$. \\
$w\left(r_{2}\right)$ & $=0.99962 \ldots$ & $=0.99899 \ldots$ & $=1.03438 \ldots$ & $=0.99259 \ldots$ \\
$d_{111}$ & $=0.90689 \ldots$ & $=0.90689 \ldots$ & $=0.90689 \ldots$ & $=0.90689 \ldots$ \\
$d_{112}$ & $=0.91058 \ldots *$ & $=0.91384 \ldots *$ & $=0.92883 \ldots$ & $=0.93096 \ldots *$ \\
$d_{122}$ & $=0.91058 \ldots *$ & $=0.91384 \ldots *$ & $=0.93808 \ldots *$ & $=0.93096 \ldots *$ \\
$d_{222}$ & $=0.90655 \ldots$ & $=0.90598 \ldots$ & $=0.93808 \ldots *$ & $=0.90289 \ldots$ \\
\hline
\end{tabular}


Table 3

\begin{tabular}{lll}
\hline & \multicolumn{1}{c}{$A_{3}$} & \multicolumn{1}{c}{$R_{8}$} \\
\hline$r_{2}$ & $=2 \cdot \sqrt{3}-3=0.4641 \ldots$ & $=\frac{1}{3}=0.33333 \ldots$ \\
$r_{3}$ & $=2-\sqrt{3}=0.2679 \ldots$ & $=\frac{1}{6}=0.16666 \ldots$ \\
$w\left(r_{1}\right)$ & $=1$. & $=1$. \\
$w\left(r_{2}\right)$ & $=1.03$ & $=1.03408 \ldots$ \\
$w\left(r_{3}\right)$ & $=1.005$ & $=0.99$ \\
$d_{111}$ & $=0.90689 \ldots$ & $=0.90689 \ldots$ \\
$d_{112}$ & $=0.92176 \ldots$ & $=0.93007 \ldots$ \\
$d_{122}$ & $=0.93021 \ldots$ & $=0.93939 \ldots$ \\
$d_{222}$ & $=0.93410 \ldots$ & $=0.93855 \ldots$ \\
$d_{113}$ & $=0.93350 \ldots$ & $=0.94756 \ldots$ \\
$d_{123}$ & $=0.93650 \ldots *$ & $=0.94882 \ldots *$ \\
$d_{133}$ & $=0.93626 \ldots$ & $=0.94882 \ldots *$ \\
$d_{223}$ & $=0.93509 \ldots$ & $=0.94007 \ldots$ \\
$d_{233}$ & $=0.92822 \ldots$ & $=0.92823 \ldots$ \\
$d_{333}$ & $=0.91143 \ldots$ & $=0.89783 \ldots$ \\
\hline
\end{tabular}

are equal is provided by the solution of a system of linear equalities in each case. It can be easily checked that for the actual coefficients the order of the other densities does not change in the neighborhood of the solution.

$R_{4}$

In packing $R_{4}$ a pair of adjacent small circles is surrounded by a ring of six unit circles so that each small circle is in contact with one small and four large ones and each large circle is in contact with three large circles and four small ones. The radius $r_{2}$ is defined by the equation

$$
\arccos \frac{r_{2}}{1+r_{2}}+3 \cdot \arcsin \frac{1}{1+r_{2}}=\pi .
$$

The whole packing is a (doubly) periodic tessallation whose fundamental domain is the convex hull of the centers of six unit circles (Fig. 4). The tight triangles are of types $t_{112}$ and $t_{122}$.

To prove the validity of condition (ii) we consider the decomposition of the packing into marked tight triangles and remove a finite set of them. We have to show that any properly marked filling of this hole with triangles of types $t_{112}$ and $t_{122}$ reproduces the original decomposition.

Let us notice that in a properly marked tiling both kinds of tiles must be present around each small circle since on the one hand four sectors of $t_{112}$ tiles are not enough to make up a small circle and five are too many, on the other hand no small circle can be covered by just $t_{122}$ tiles since they can occur only in pairs joined along their base, and two pairs are not enough while three are too many. It is easy to see that the configuration around every small circle must be the same: three $t_{112}$ tiles in sequence then a close pair of $t_{122}$ tiles. Consequently, in every properly 


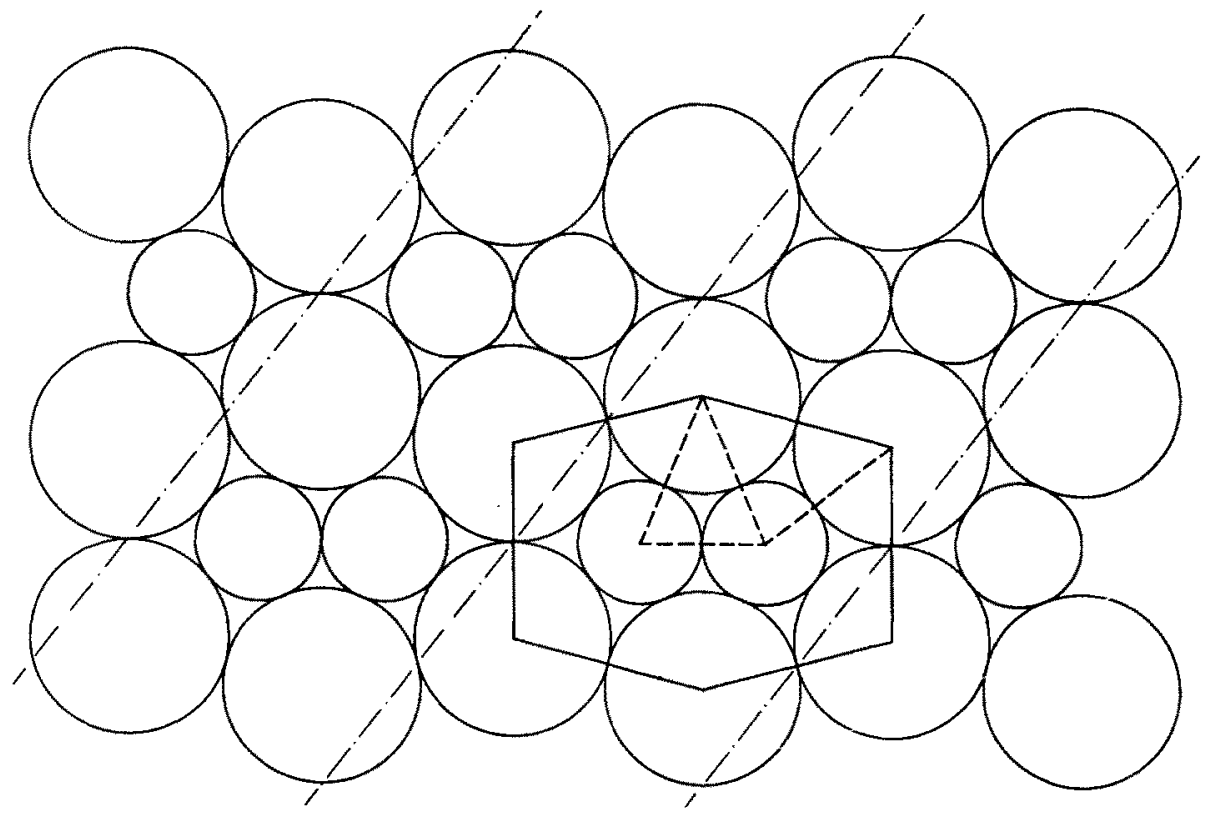

Fig. 4.

marked tiling with tight triangles of types $t_{112}$ and $t_{122}$ the tiles can be grouped into disjoint hexagonal blocks described above.

Knowing this, first we enlarge the hole $U$ to be the union of several such blocks and consider a new filling of the hole with marked tight triangles of maximal density so as to obtain a proper tiling of the whole plane. As the length of the line segments (sides of triangles) cutting a hexagon into tiles are all different from 2 , the hexagonal blocks of the new tiling do not "cross" the boundary of the hole.

Now all we have left to show is that the hole can be filled with fundamental hexagons only in one way. Let $L$ be a straight line through the centers of two adjacent small circles of the original pattern and let $C$ be a corner of the hole lying on a support line parallel to $L . C$ is a corner of the largest angle of a single hexagon of the original decomposition of the hole while the other hexagons meeting in $C$ lie outside of the hole. This corner of the hole, however, can be filled with fundamental hexagons in a unique way (by a single hexagon) and that coincides with the original pattern. Thus, in a finite number of steps the original pattern is reproduced.

$R_{5}$

Each small circle is surrounded by a ring of three unit circles and two small ones in $R_{5}$ in such a way that the small circles are separated by the unit ones. The unit circles have eight neighbors: three small circles, a unit one, three small, and again a unit one. The whole packing is a periodic repetition of a fundamental parallelo- 


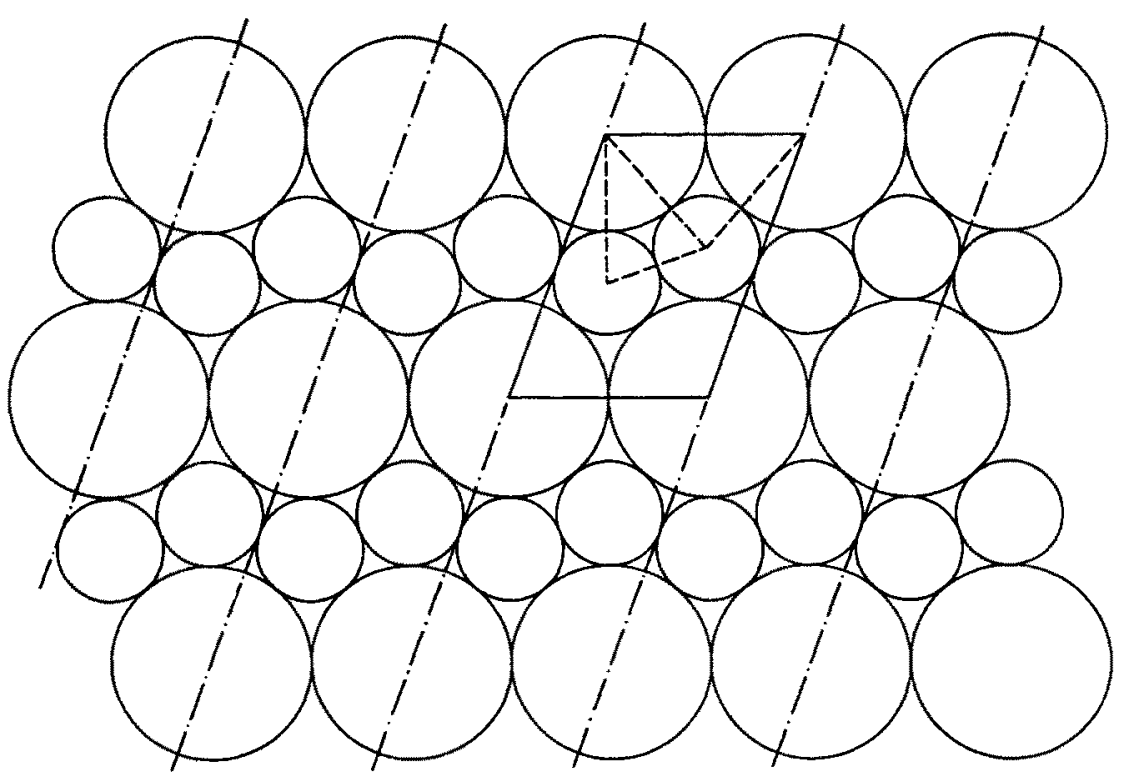

Fig. 5

gram (that breaks down into two isosceles triangles) containing sectors of a unit circle at the corners and two complete small circles inside (Fig. 5). Thus the neighboring unit circles are arranged into straight strings. The size of the small circle is defined by the equation

$$
2 \cdot \arccos \frac{r_{2}}{1+r_{2}}+\arcsin \frac{1}{1+r_{2}}=\pi
$$

The tile triangles are of types $t_{112}$ and $t_{122}$. The validity of condition (ii) can be shown with a similar argument to that of the case of $R_{4}$. It can also be proved that in any proper tiling the triangles can be grouped to form rhombs (two kinds of them). Then our problem reduces to filling a-suitably enlarged-hole with these two kinds of marked rhombs.

$R_{6}$

This packing can also be derived from the Archimedean tessallation $(3,12,12)$ with sidelength $2 \cdot r_{2}$. Instead of taking the incircles of the polygons as we did to get $A_{1}$ we set circles of radius $r_{2}$ around the corners and draw unit circles concentric to the dodecagons (Fig. 6). The value of $r_{2}$ is defined by the equality

$$
12 \cdot \arcsin \frac{r_{2}}{1+r_{2}}=\pi
$$




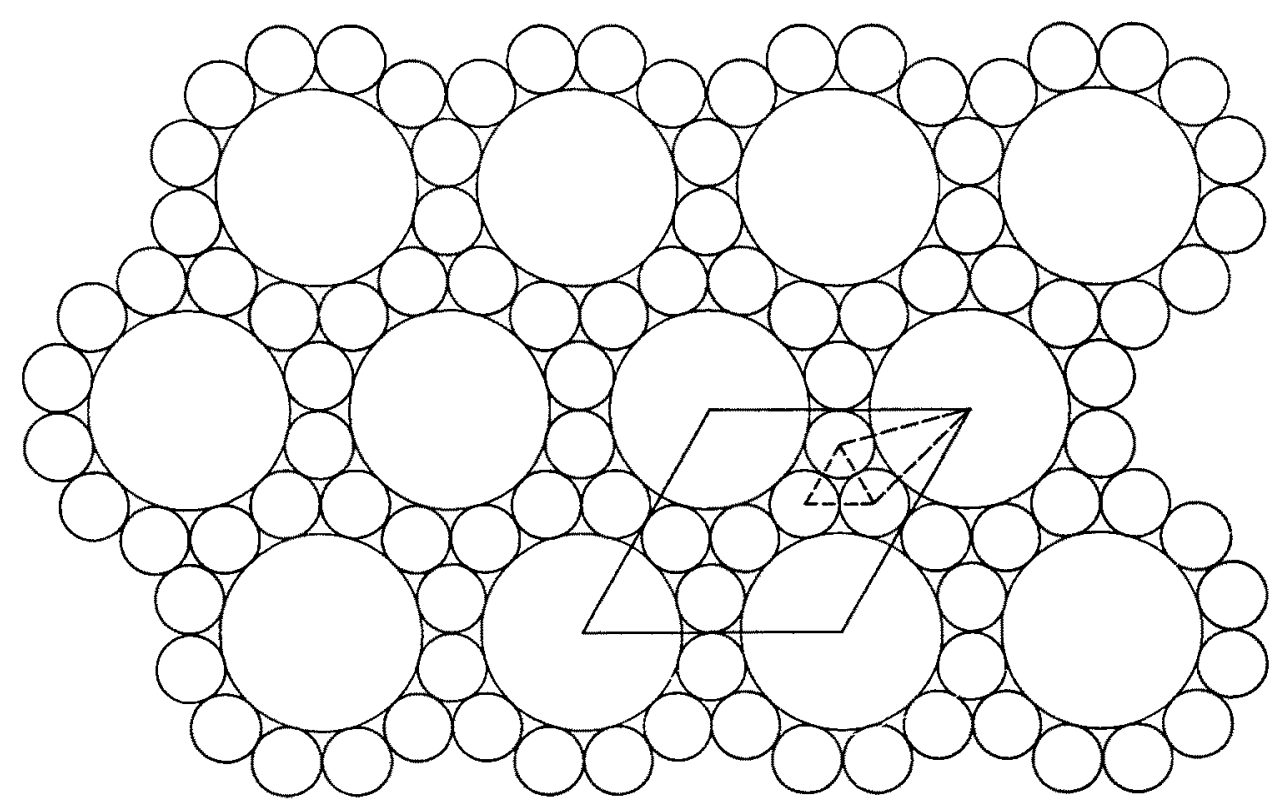

Fig. 6

The tile triangles of this packing are of type $t_{222}$ and $t_{122}$. Sidelength $\left(1+r_{2}\right)$ occurs only in tile $t_{122}$, thus in every properly marked tiling the $t_{122}$ tiles must be arranged to form regular dodecagons of sidelength $2 \cdot r_{2}$. Let us now remove a finite set of triangles from the original decomposition then enlarge this hole until no dodecagonal block belongs partly to the hole and partly to the rest of the decomposition. Then the boundary of the hole consists of segments of length $2 \cdot r_{2}$. The inner angles of the hole at the corners of the convex hull of the hole clearly show whether a $t_{222}$ tile or a dodecagonal block fills this corner properly.

$R_{7}$

This packing is defined through the periodic arrangement of rhombs of sidelength 2. The unit circles around the vertices of a rhomb enclose a pair of touching small circles (Fig. 7). Radius $r_{2}$ of the small circle is defined by the equality

$$
\arccos \frac{r_{2}}{1+r_{2}}+2 \cdot \arcsin \frac{1}{1+r_{2}}=\pi
$$

The tile triangles are of type $t_{112}$ and $t_{122}$. The proof of the solidity of the tiling is similar to that of packing $R_{4}$. The hole is enlarged to be the union of rhombs congruent to the fundamental domain, and the original problem is reduced to that of filling the hole with marked rhombs. 


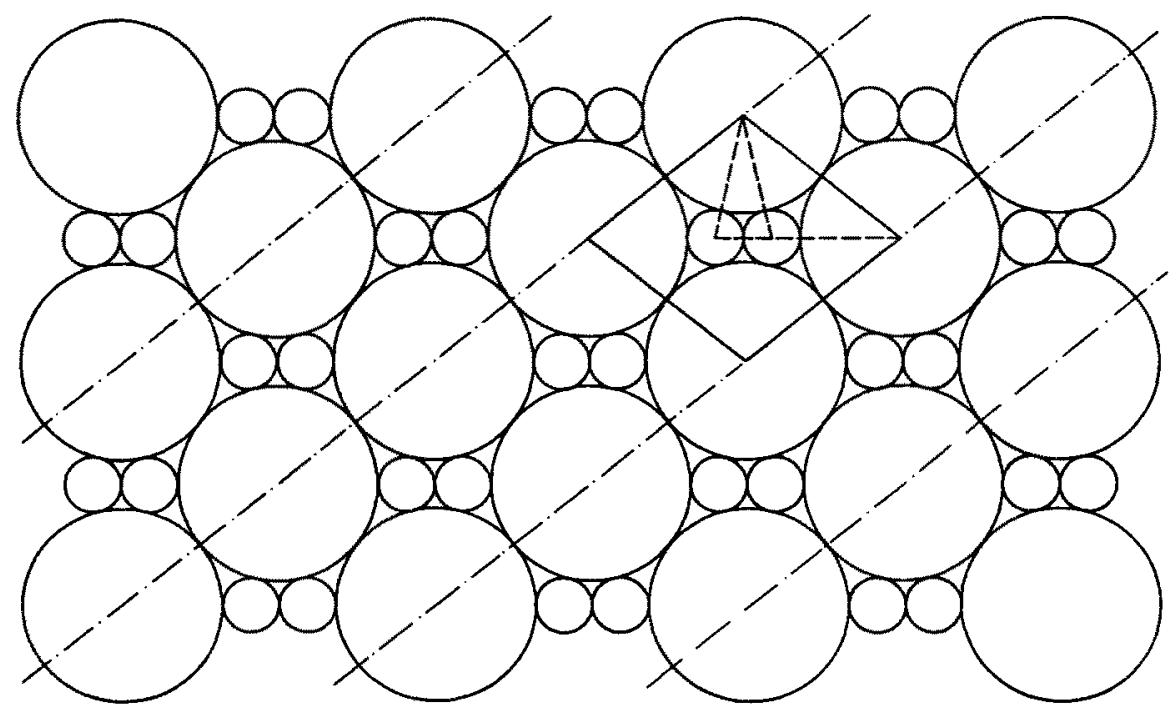

Fig. 7

$R_{8}$

In this packing there are three different circles, and two different tiles. The radii are

$$
r_{2}=\frac{1}{3}=0.33333 \ldots, \quad r_{3}=\frac{1}{6}=0.16666 \ldots,
$$

and the tiles are of types $t_{123}$ and $t_{133}$. The unit circles are concentric with the hexagons of a regular hexagonal tessallation each surrounded by six circles of medium and twelve of smallest size (Fig. 8).

Considering the angles of the tiles it is easy to see that in any proper tiling the unicity of certain sidelengths of the tiles implies that the tiles must form regular hexagonal blocks. To prove that condition (ii) holds, first the hole is enlarged until it is the union of regular hexagons of sidelength $\frac{4}{3}$. The filling of this hole with regular hexagons of this size is possible only along the original pattern.

\subsection{Other Arrangements}

Let us note that in some cases more than one proper tiling can be obtained using densest marked tiles. Consider, e.g., the tiling generated by packing $R_{7}$, the marked strip $T$ consisting of the infinite sequence of fundamental rhombs lying between two parallel straight lines $L$ and $L^{\prime}$ bounding such a rhomb, finally, the reflection $\mathrm{T}^{\prime}$ of $T$ in $L$. A proper tiling can be formed by joining translated copies of $T$ and $T^{\prime}$ in any order. Thus, changing the order, an infinite variety of proper tilings-family $F_{7-7}-$ can be constructed (Fig. 9). The corresponding "layered" packings have one-dimensional periodicity. It is easy to see that all proper tilings using the extremal marked triangles $t_{112}$ and $t_{122}$ as building units belong to family $F_{7-7}$, and they are all uniquely determined. 


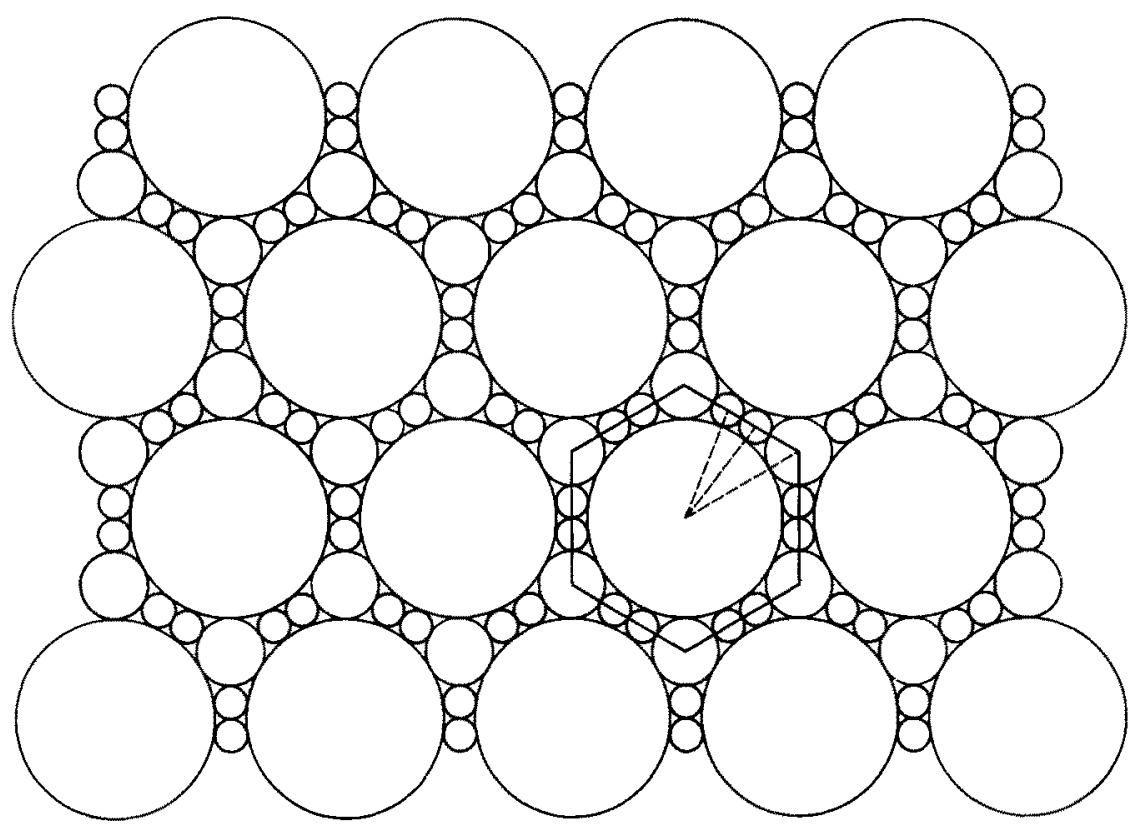

Fig. 8

The "layered" packings of family $F_{4-4}$, and $F_{5-5}$, - generated from $R_{4}$ and $R_{5}$ in a similar way-are also solid and have one-dimensional periodicity. For $F_{4-4}$ ' consider the convex hull of the side midpoints of the fundamental hexagon. The fundamental strip is defined by two opposite sides of this hexagon-not parallel to the line connecting the centers of two touching small circles (Fig. 4). For $F_{5-5}$, the fundamental strip is bounded by the common tangent line of two touching small circles of $R_{5}$ and the nearest such line parallel to the first one (Fig. 5).

Another way to generate solid packings is to combine strips of different solid packings. In families $F_{0-2}, F_{1-7-7}, F_{1-5}$, and $F_{2-5}$ the strips are bounded by

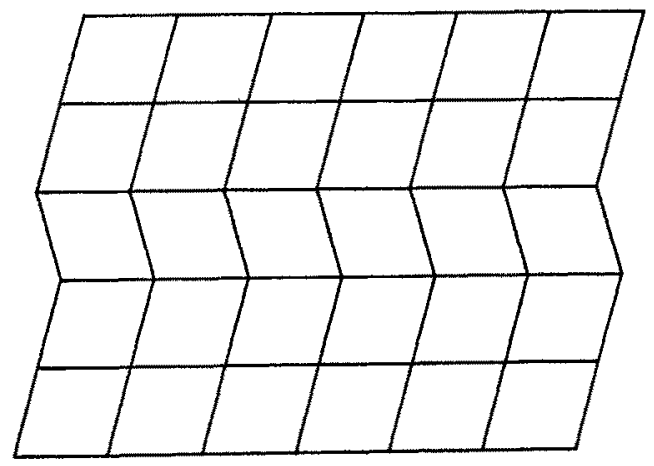

Fig. 9 


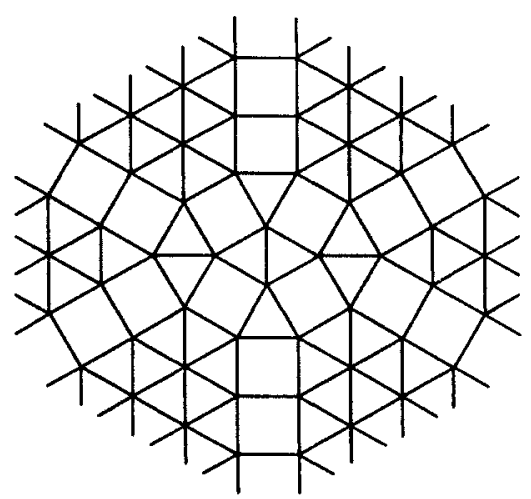

Fig. 10

straight lines connecting the centers of unit circles in contact taken from packings $R_{0}$ and $A_{2}, A_{1}$ and $R_{7}, A_{1}$ and $R_{5}, A_{2}$ and $R_{5}$, respectively, where $R_{0}$ denotes the densest lattice packing of unit circles. These packings are all solid.

The marked triangles used in family $F_{\mathrm{O}_{-2}}$ provides even more freedom in constructing compact packings. Since for $r_{2}=\sqrt{2}-1$ and for suitably chosen weights $d_{111}=d_{112}$ holds, all marked tilings formed from marked squares and regular triangles of sidelength 2 fulfill condition (i), therefore the corresponding packings are-at least-weakly solid. Besides the family $F_{0-2}$ of all layered packings mentioned above there exist examples of solid packings with no periodicity (Fig. 10) as well as examples of centrally symmetrical packings that are not solid (Fig. 11(a) and (b)).

Finally, although the layered tilings based on strips taken from packings $A_{2}$ and $R_{7}$ are uniquely determined (condition (ii)), the solidity of the corresponding packings is not guaranteed since condition (i) does not hold. This example shows that conditions (i) and (ii) are independent.

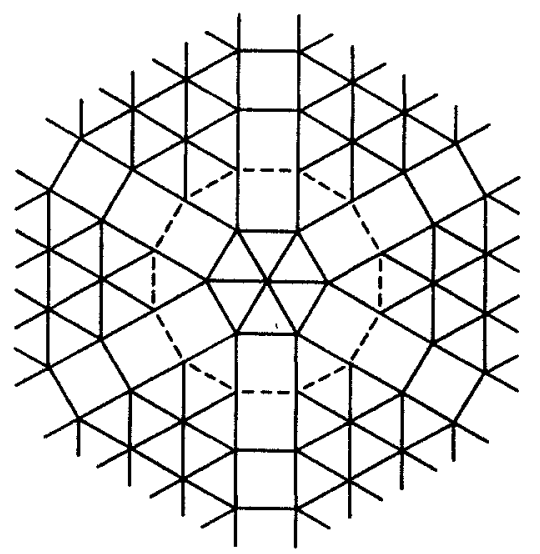

(a)

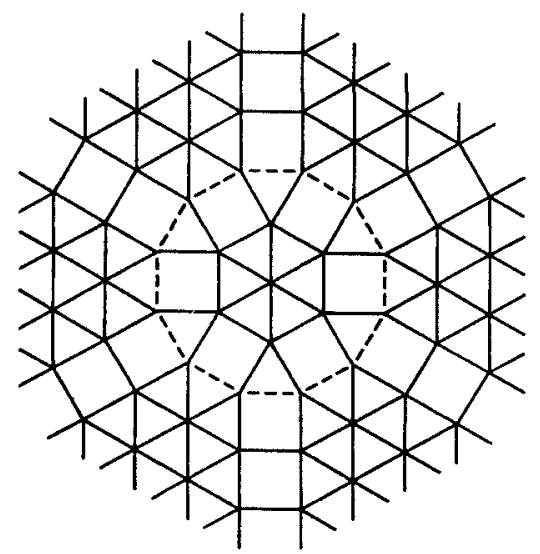

(b)

Fig. 11 


\section{Corollary}

We begin with some definitions. The number-density $v(r)$ of a packing of circles of radius $r$ can be defined as $1 /\left(\pi \cdot r^{2}\right)$ times its (common) density. In a packing of circles of radius $r_{1}, r_{2}, \ldots, r_{k}$ let $\lambda\left(r_{i}\right)=v\left(\mathrm{r}_{\mathrm{i}}\right) / v\left(r_{1}\right)$ be the relative number-density of circles of radius $r_{i}$ and radius $r_{1}(i>1)$. We conclude this paper by directing the attention of the reader to the simple fact that if a packing of circles of radii $r_{1}$, $r_{2}, \ldots, r_{k}$ is (weakly) solid, then it has maximal density among those packings which consist of the same kind of circles having the same relative number-densities.

\section{Acknowledgment}

The author wishes to thank the referees for greatly improving the readability of this paper.

\section{References}

1. A. Bezdek, Solid packing of circles in the hyperbolic plane, Studia Sci. Math. Hungar. 14 (1979), 203-207.

2. G. Fejes Tóth, Solid sets of circles, Studia Sci. Math. Hungar. 9 (1974), 101-109.

3. L. Fejes Tóth, Solid circle-packings and circle-coverings, Studia Sci. Math. Hungar. 3 (1968), 401-409.

4. L. Fejes Tóth, Compact packing of circles, Studia Sci. Math. Hungar. 19 (1984), 103-107.

5. L. Fejes Tóth and J. Molnár, Unterdeckung und Uberdeckung der Ebene durch Kreise, Math. Nachr. 18 (1958), 236-243.

6. A. Florin, L. Hárs, and J. Molnár, On the $\rho$-system of circles, Acta Math. Acad. Sci. Hungar. 34 (1979), 205-221.

7. B. Grünbaum and G. C. Shephard, Tilings and Patterns, Freeman, San Franciso, 1987.

8. A. Heppes and J. Molnár, Ujabb eredmények a diszkrét geometriában I, Mat. Lapok XI (1960), 330-355.

Received June 16, 1989, and in revised form February 28, 1990. 